\title{
ON THE SATURATION OF ASTROPHYSICAL DYNAMOS: NUMERICAL EXPERIMENTS WITH THE NO-COSINES FLOW
}

\author{
S. B. F. DORCH ${ }^{1}$ and V. ARCHONTIS ${ }^{2}$ \\ ${ }^{1}$ The Niels Bohr Institute for Astronomy, Physics and Geophysics, Juliane Maries Vej 30, \\ Copenhagen $\emptyset$, Denmark \\ (e-mail: dorch.astro.ku.dk) \\ ${ }^{2}$ Instituto de Astrofisica de Canarias, Via Lactea, La Laguna, Spain
}

(Received 31 August 2004; accepted 19 October 2004)

\begin{abstract}
In the context of astrophysical dynamos we illustrate that the no-cosines flow, with zero mean helicity, can drive fast dynamo action and we study the dynamo's mode of operation during both the linear and non-linear saturation regimes. It turns out that in addition to a high growth rate in the linear regime, the dynamo saturates at a level significantly higher than normal turbulent dynamos, namely at exact equipartition when the magnetic Prandtl number $\operatorname{Pr}_{\mathrm{m}} \sim 1$. Visualization of the magnetic and velocity fields at saturation will help us to understand some of the aspects of the non-linear dynamo problem.
\end{abstract}

\section{Introduction}

Magnetic fields are common in astronomical objects and are responsible for a variety of complex phenomena in the universe. It is widely accepted that these fields are generated by the motions of conducting fluids through a transfer of kinetic energy to magnetic energy, that is, dynamo action (Parker, 1979). However, our understanding of the exact mechanism of generation and maintenance of the magnetic field against dissipation is still incomplete even for simple dynamos. On the observational side, when a magnetic field in an astronomical object is not observed directly often the inferred equipartition value $B_{\text {eq }}=u \sqrt{\mu_{0} \rho}$ (where $u$ and $\rho$ are characteristic speed and mass density, and $\mu_{0}$ is the vacuum permeability) is assumed, e.g., Safier (1999). Therefore it is of importance to consider whether this is a good approach. In the Sun the equipartition field strength at the surface is about 500 gauss. However, the mean magnetic field strength near the Sun's polar regions has a canonical value of 1-5 G going back to Babcock, which is far less than $B_{\text {eq }}$; additionally, the intermittent flux tubes at the surface are much stronger, with field strengths around $1-3 \mathrm{kG}$ in spots and flux tubes, while it is inferred to be $10 \mathrm{kG}$ near the bottom of the convection zone, e.g., Moreno-Insertis, Caligari, and Schüssler (1994). In any case, $B_{\text {eq }}$ is not a good estimate for the solar magnetic field. Furthermore, on the theoretical side of things, numerical models of turbulent non-linear dynamo action as a rule produce strong intermittent structures, but cannot be said to generally saturate at equipartition; these models can be used to study the generic properties of real astrophysical dynamos, such as the solar dynamo (Choudhuri, 2003). 
Dynamo action in the limit of infinite magnetic Reynolds number $\operatorname{Re}_{\mathrm{m}}=u \ell / \eta$ (where $\ell$ is a characteristic length scale and $\eta$ is the magnetic diffusivity) is relevant to astrophysical systems where the diffusion timescale is larger than the advection timescale, such as in the Sun. Considering the importance of the magnetic forces relative to the motion of the fluid, one divides dynamo action into two regimes: the linear kinematic regime in which the flow amplifies the magnetic field exponentially by, e.g., stretching the magnetic field lines (Childress and Gilbert, 1995), and the non-linear regime where the magnetic field becomes strong enough to modify the initial flow topology through the Lorentz force and saturate the exponential growth.

Recent studies (Dorch, 2000; Archontis, Dorch, and Nordlund, 2003a; Tanner and Hughes, 2003) have improved our knowledge of fundamental dynamo mechanisms of prescribed flows in the kinematic regime. Non-linear fast dynamo action has received considerable attention and progress has been made especially by means of numerical magneto-hydrodynamical (MHD) simulations (Nordlund et al., 1992; Cattaneo, Hughes, and Kim, 1996). However, it is still unclear what the physical processes at work are before and especially after the saturation of the dynamo. It is generally found that dynamos saturate because of a suppression of the stretching ability of the flows (Cattaneo, Hughes, and Kim, 1996; Archontis, Dorch, and Nordlund, 2003b; Tanner and Hughes, 2003), but the details of how this comes about are not known.

In this paper, we present results from numerical experiments with a non-helical flow first studied by Galloway and Proctor (1992) that saturates below equipartition only for high values of the Reynolds number $\operatorname{Re}=u \ell / v$ ( $v$ being the viscosity). At magnetic Prandtl number $\operatorname{Pr}_{\mathrm{m}}=\operatorname{Re}_{\mathrm{m}} / \mathrm{Re} \sim 1$ saturation occurs at almost exact equipartition. We study the non-linear dynamo mode of operation, examine how it changes when the flow becomes turbulent, and take the first steps at illustrating how the fields evolve and interact.

\section{Setup of Numerical Experiments}

The fully compressible 3-d MHD equations are solved numerically on a periodic Cartesian grid using dimensionless quantities:

$$
\begin{aligned}
\frac{\partial \rho}{\partial t} & =-\nabla \cdot \rho \mathbf{u}, \\
\frac{\partial(\rho \mathbf{u})}{\partial t}+(\mathbf{u} \cdot \nabla) \rho \mathbf{u} & =-\nabla P-(\nabla \cdot \mathbf{u}) \rho \mathbf{u}+\mathbf{f}_{\mathrm{L}}+\mathbf{f}+\frac{1}{\operatorname{Re}} \rho \nabla^{2} \mathbf{u}, \\
\frac{\partial e}{\partial t} & =-\nabla \cdot(e \mathbf{u})-P \nabla \cdot \mathbf{u}+Q_{\mathrm{v}}+Q_{\mathrm{J}}+Q_{\text {cool }}, \\
\frac{\partial \mathbf{B}}{\partial t} & =\nabla \times(\mathbf{u} \times \mathbf{B})+\frac{1}{\operatorname{Re}_{\mathrm{m}}} \nabla^{2} \mathbf{B},
\end{aligned}
$$


where $\rho$ is the fluid density, $\mathbf{u}$ is the velocity, $P$ is the pressure, $\mathbf{j}$ is the electric current density, $\mathbf{B}$ is the magnetic field density and $e$ is the specific internal energy. The gas pressure $P$ is related to the density $\rho$ through an ideal equation of state. In Equation (2) $\mathbf{f}_{\mathrm{L}}=\mathbf{j} \times \mathbf{B}$ is the Lorentz force per unit volume giving rise to an increase or decrease in the total magnetic energy, depending on the sign of the power term $W_{\mathrm{L}}=\int_{\mathrm{V}} \mathbf{u} \cdot \mathbf{f}_{\mathrm{L}} \mathrm{dV}$. In the same units $Q_{\mathrm{v}}$ and $Q_{\mathrm{J}}$ are the viscous and Joule dissipation, respectively. $Q_{\text {cool }}=\left(T-T_{0}\right) / \tau_{\text {cool }}$ is a Newtonian cooling term with $T_{0}$ and $\tau_{\text {cool }}=5$ being the reference temperature and cooling time, respectively. The cooling term works as a sink of the thermal energy in order to balance the heat variations in the energy equation. $\mathbf{f}=-K / \operatorname{Re} \nabla^{2} \mathbf{u}_{0}$ ( $\mathbf{u}_{0}$ being defined below) is an external prescribed forcing term with an amplitude $K$ that is allowed to evolve with time, keeping the average kinetic energy approximately constant through both laminar and turbulent phases, cf., Archontis, Dorch, and Nordlund (2003b).

Equations (1) - (4) are solved numerically on a staggered mesh using derivatives and interpolations that are of 6th and 5th order respectively, in a numerical scheme that conserves $\nabla \cdot \mathbf{B}=0$ exactly. Numerical solutions are obtained on a grid of $128^{3}$ points, using the code by Nordlund and others (Nordlund et al., 1992).

The chosen initial velocity field is similar to the classical ABC flow (Dorch, 2000) but does not contain cosine terms:

$$
\mathbf{u}_{0}=(\sin z, \sin x, \sin y)
$$

where the coordinates $(x, y, z)$ have a periodicity of $2 \pi$ in all directions: Hence the characteristic scales are $\ell=2 \pi$, and $u=1$. The total kinetic helicity $\int_{V} \mathbf{u}$. $(\nabla \times \mathbf{u}) d V$ (where $\nabla \times \mathbf{u}$ is the vorticity) is identically zero for this flow that has previously been found to be a good candidate for fast dynamo action. The growth rate of the magnetic field is known to increase as a function of $\mathrm{Re}_{\mathrm{m}}$ in the linear regime, at least until $\mathrm{Re}_{\mathrm{m}}=800$ (Galloway and Proctor, 1992). The initial magnetic seed field is chosen to be weak and divergence-free with an amplitude of $10^{-5}$ in non-dimensional units.

\section{Results and Discussion}

We have performed several numerical experiments with varying magnetic Reynolds number $\mathrm{Re}_{\mathrm{m}}$ between 50-200 and kinetic fluid Reynolds number Re between 2-450.

Initially during the linear, kinematic regime the total magnetic energy $E_{\mathrm{mag}}$ grows exponentially because of a small difference between the contribution $W_{\mathrm{L}}$ through the Lorentz force and the Joule dissipation $Q_{\mathrm{J}}$. Figure 1 shows the evolution of total energy in an experiment with $\mathrm{Re}_{\mathrm{m}}=100$ and $\mathrm{Re}=2$. In this case the magnetic field was initially given by a weak uniform seed field and this special topology results in several linear dynamo modes being exited, but eventually $E_{\text {mag }}$ saturates at equipartition within a few fractions of a percent. 


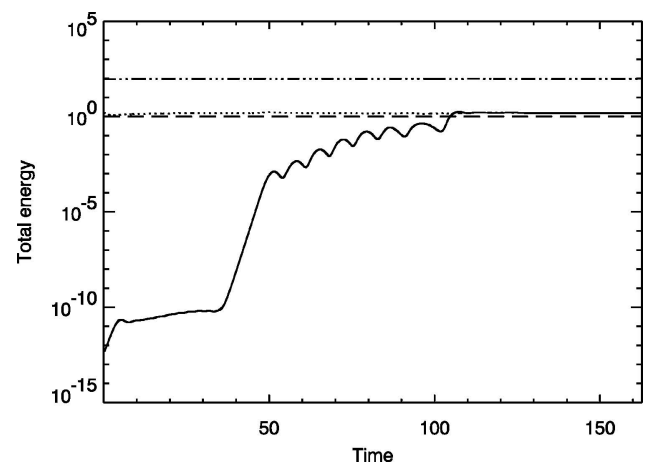

Figure 1. Total energies as a function of time for an experiment with $\operatorname{Re}_{\mathrm{m}}=100$ and $\mathrm{Re}=2$. Here the solid curve is magnetic energy $E_{\mathrm{mag}}$, the dotted curve kinetic energy $E_{\mathrm{kin}}$ and the dotted-dashed curve the total thermal energy $E_{\mathrm{th}}$. The dashed horizontal line indicates unity energy.

The balance between $W_{\mathrm{L}}$ and $Q_{\mathrm{J}}$ in the linear regime does not hold in places with weak magnetic field and little dissipation, as has also been shown in previous kinematic dynamo experiments (Archontis, Dorch, and Nordlund, 2003a). A calculation, for low $\mathrm{Re}$, of the average net power $-W_{\mathrm{L}}-Q_{\mathrm{J}}$ shows that most of the energy growth responsible for the fast dynamo action occurs in places with less than $20 \%$ of the maximum dissipation and magnetic field strength and occupies $98 \%$ of the volume. There, the net power is $95 \%$ of the maximum level. In the remaining $2 \%$ of the volume the strong dissipation is balanced by the energy input resulting from the work done on the field by the flow through advection and convergence.

If the magnetic field is initialized by a random seed field fewer linear modes appear before saturation: Figure 2 (left) shows magnetic energy as a function of time for a case with $\mathrm{Re}_{\mathrm{m}}=100$ and $\mathrm{Re}=50$ that also saturates near equipartition, but only has one dominant, very fast growing mode during the linear regime.
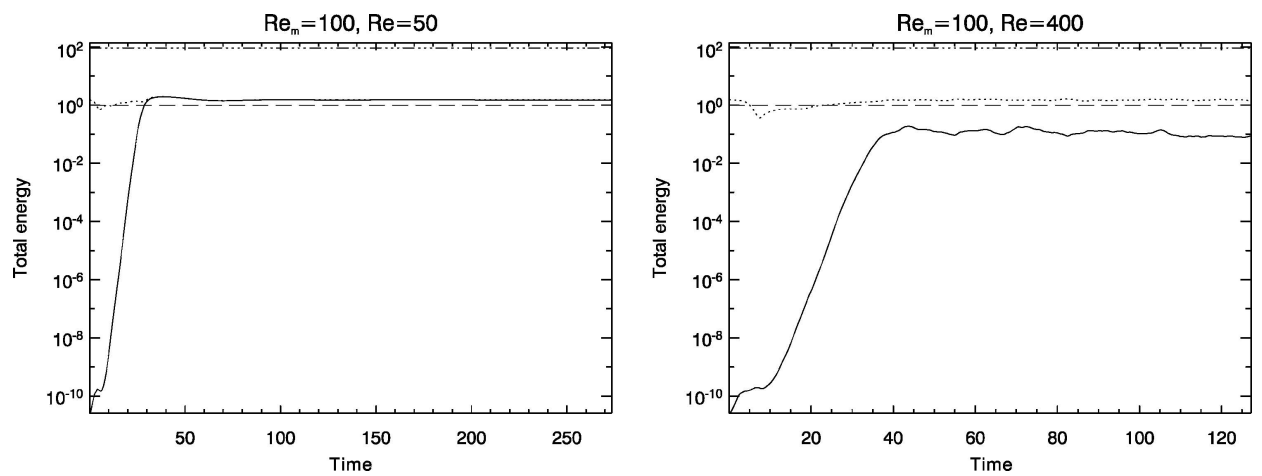

Figure 2. Total magnetic, kinetic and thermal energy as functions of time for cases with $\operatorname{Re}_{\mathrm{m}}=100$ and $\operatorname{Re}=50$ (left) and $\operatorname{Re}_{\mathrm{m}}=100$ and $\mathrm{Re}=400$ (right). Line styles are the same as in Figure 1. 

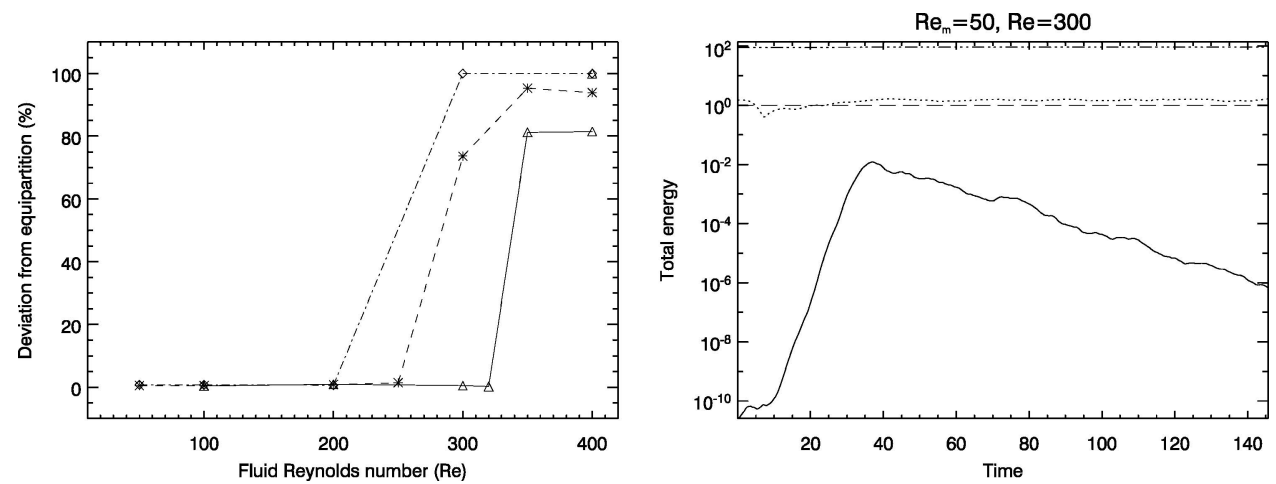

Figure 3. Left: A summary figure of the deviation (in percent) from saturation at exact equipartition as a function of fluid Reynolds number Re. Three regimes are shown corresponding to experiments with $\operatorname{Re}_{\mathrm{m}}=200$ (full curve), $\mathrm{Re}_{\mathrm{m}}=100$ (dashed curve) and $\mathrm{Re}_{\mathrm{m}}=50$ (dashed-dotted curve). Right: Total magnetic, kinetic and thermal energy as functions of time for an experiment with $\mathrm{Re}_{\mathrm{m}}=50$ and $\operatorname{Re}=300$ corresponding to $\operatorname{Pr}_{\mathrm{m}}=1 / 6$. Line styles are the same as in Figure 1 .

In these cases where Re is low, the flows are very laminar, as we shall show below, but if Re is increased turbulence sets in. With well-developed turbulence the magnetic energy saturates significantly below equipartition as can be seen in Figure 2 (right) for a case also with $\mathrm{Re}_{\mathrm{m}}=100$ but $\mathrm{Re}=400$, where in the non-linear regime $E_{\mathrm{mag}} \approx 0.2 E_{\mathrm{kin}}$. This dependence on Re is summarized in Figure 3 (left) that shows the deviation from exact equipartition, after saturation, as a function of $\operatorname{Re}$ for three different values of $\operatorname{Re}_{\mathrm{m}}$. Apparently there is a jump at $\operatorname{Re} \sim 300$ below which the dynamo saturates at exact equipartition. This scenario can also be quantified in terms of magnetic Prandtl number $\operatorname{Pr}_{\mathrm{m}}$. There is a critical value $\operatorname{Re}^{(c)}$ below which the dynamo saturates below equipartition and the value of $\operatorname{Re}^{(\mathrm{c})}$ depends on $\operatorname{Pr}_{\mathrm{m}}$ roughly as $\operatorname{Re}^{(\mathrm{c})} \sim 50 \mathrm{Pr}_{\mathrm{m}}^{0.5}$. At sufficiently low $\operatorname{Pr}_{\mathrm{m}}$ the dominating dynamo mode is in fact a decaying mode, see e.g. Figure 3 (right) that shows the behavior of the total energy for a case with $\operatorname{Re}_{\mathrm{m}}=50$ and $\mathrm{Re}=300$ corresponding to $\operatorname{Pr}_{\mathrm{m}}=1 / 6$.

A tell-tale result is that the flow (for low Re), after it goes through a turbulent phase just prior to saturation, comes out as a laminar solution with the magnetic and velocity field parallel and equal in magnitude, see Figure 5 (left): e.g. for the experiment with $\mathrm{Re}_{\mathrm{m}}=200$ and $\mathrm{Re}=200$ less than $2 \%$ of the volume is occupied by field and flows inclined by more than $10^{\circ}$. In this case, with unit magnetic Prandtl number $\operatorname{Pr}_{\mathrm{m}}$, the viscous and resistive losses are both directly proportional to the velocity and magnetic field strength, respectively, with the same constant of proportionality and equal to $1 / \operatorname{Re}$. For $\operatorname{Pr}_{\mathrm{m}} \neq 1$ the two fields are still proportional but not exactly equal and the saturation level deviates from exact equipartition in fractions of a percent.

In fact, there are marked differences between the structure of the magnetic and velocity fields in the experiments as $\mathrm{Re}$ is increased: This is true both in Fourier 

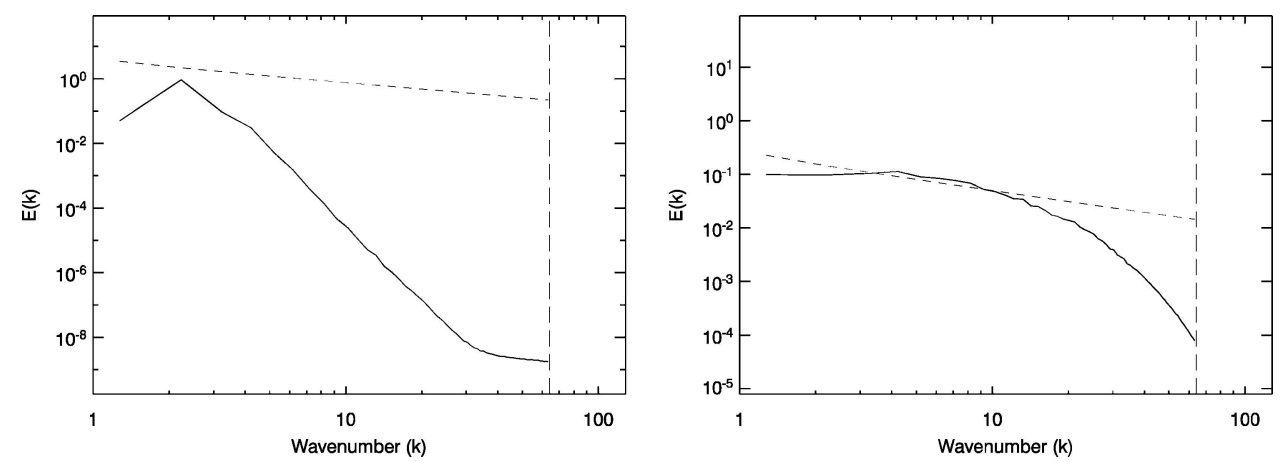

Figure 4. Energy spectra of magnetic energy as function of wavenumber $k$ for for a case with $\mathrm{Re}_{\mathrm{m}}=$ 50 and $\mathrm{Re}=200$ (left), and a case with $\mathrm{Re}_{\mathrm{m}}=200$ and $\mathrm{Re}=450$ (right). The vertical dashed lines correspond to the numerical resolution limit (the Nyquist frequency) and the inclined straight lines indicate a power law with exponent $-5 / 3$ (Kolmogorov scaling).

$k$-space as well as in physical space. In $k$-space, for $\operatorname{Re}_{\mathrm{m}}=200$ and $\mathrm{Re}=200$, the magnetic and kinetic energy power are completely coincident (with a ratio of $\sim 0.95$ ) except at the smallest scales where there is slightly more power in the flow. The magnetic energy has a maximum at $k \approx 2.2$ and the spectra approximately follow a power-law with a power of -3 , but for $k \geq 10$ become very steep, see Figure 4 (left). In cases with $\mathrm{Re}_{\mathrm{m}}=200$ and higher Re there is also a lot of power at large scales, but the spectrum is much flatter and roughly follows Kolmogorov scaling for intermediate wavenumbers, see Figure 4 (right). Examination of the magnetic energy per wavenumber $k \mathrm{E}(k)$ reveals that most energy comes from scales $k \approx 10$ similar to what was found in models of non-helical turbulence by Haugen, Brandenburg, and Dobler (2003).

In physical space, the behavior of the magnetic field and the velocity flow can be quiet revealing. Hereafter, we consider first the situation with $\mathrm{Re} \leq 200$ and then a higher $\mathrm{Re}=400$ case. In both cases, and during the exponential amplification of the magnetic energy, the magnetic field has the form of strong sheets or tubes which are spiraling around strong vortex tubes. The weak background field is stretched by the flow and is folded against the sheets in a highly twisted manner. The situation is dramatically changed when the exponential amplification of the magnetic energy stops. The saturation regime for the $R e=200$ case shows that the flow and the magnetic field have a less complex configuration and consist of regions with stagnation points. Figure 5 (left) illustrates how two different type of stagnation points (marked as "a" and " $b$ " in the figure) connect each other. Streamlines from the a-type points are converging along an axis towards the center of the computational domain, where a b-type point is located, and then diverge in a spiral manner. This is also the topology of the flow at the beginning of the simulation. The difference with the growth phase is that now there are magnetic null-points that coincide with flow stagnation points. We note that the importance 

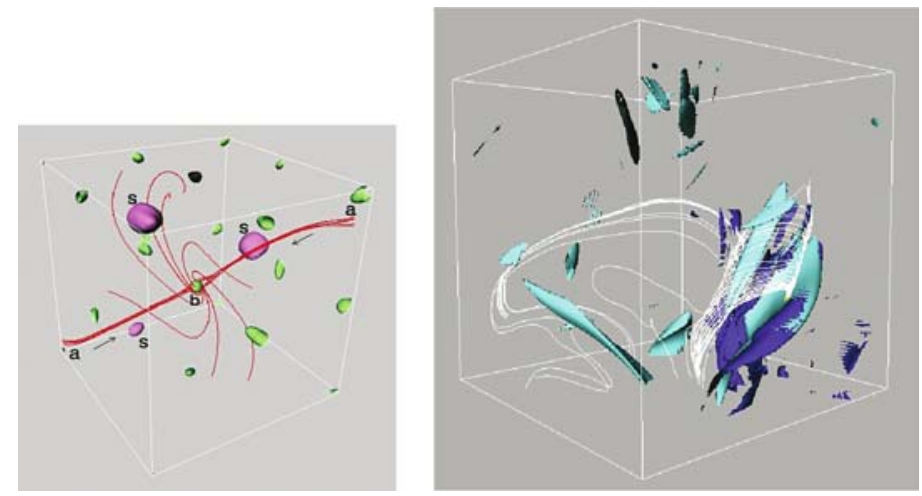

Figure 5. Left: Volume rendering of the magnetic field and velocity flow in the experiment with $\mathrm{Re}_{\mathrm{m}}=200$ and $\mathrm{Re}=200$ during saturation. Isosurfaces of regions of low magnetic field and flow, and local maxima marked with "s", are shown. The lines are magnetic field lines and the arrows show their direction. Right: Strong magnetic field (light) and vorticity structures (dark) in the experiment $\mathrm{Re}_{\mathrm{m}}=200$ and $\mathrm{Re}=400$ during saturation. Field lines traced from weak magnetic field (left side of the box) are stretched and pile up in twisted sheets around strong vortex tubes (to the right).

of the existence of the stagnation points is that the stretching of the fieldlines is maximum in their neighborhood. Also, the magnetic and velocity fields take the form of collapsed "blobs" of parallel (as a natural consequence of equipartition) field and stream lines that have been folded and intensified. Next to these are sheets of strong vorticity (not shown in the figure for clarity reasons).

In the case of the experiments with high Re, the magnetic energy saturates well below equipartition, with more fluctuations than in the $\mathrm{Re}=200$ case and timeintervals where $E_{\mathrm{mag}}$ increases or decreases (Figure 2, right). During the increase most of the power again comes from the regions between the strong magnetic structures where dissipation is low. The velocity has still a good grip on the weakest part of the field and increases the magnetic energy by stretching the field lines. The magnetic field has the form of intermittent flux tubes or sheets that are twisted around regions of high vorticity. Figure 5 (right) shows how fieldlines, which are traced from the left side of the domain in place where the magnetic field is weak, form long stretched loops and pile up against strong flux sheets that whirl around high vorticity structures in the right side of the domain. The twisting of the magnetic field increases the tension of the magnetic field lines and thereby the magnetic energy. Twisted sheets with like polarity merge and those with opposite polarity reconnect (reconnection is essential for the replenishment of the weak background field), with the most rapid release of energy occurring when $E_{\text {mag }}$ is maximum.

During the decrease of the magnetic energy the twisted magnetic structures split into smaller pieces, which are now connected each other with straight rather than with twisted field lines. The above mode is characteristic of the kinematic $\mathrm{ABC}$ dynamo experiments where stretch, twist, fold and reconnection of fieldlines between magnetic flux cigars drive a periodic exponential growth of the magnetic 
energy (Dorch, 2000). In the present non-linear case however, there is no periodic behavior, the flow has clearly deviated from its initial configuration (in contrast also with the $R e=200$ case where the flow returns to its initial topology) and the dynamo mode of operation is a symmetry-breaking mode.

\section{Conclusion}

We have presented results from new numerical experiments with non-linear dynamo action by a non-helical flow while varying characteristic dimensionless parameters. The stretching of the weak part of the magnetic field seems to be the main dynamo mechanism during the different phases of the simulation (growth phase, saturation phase, linear and/or turbulent regime). 3-d visualization of the flow and the magnetic field shows similar structures associated with stretching, folding and subsequent amplification of the magnetic energy, in both the linear and non-linear equilibrated regime. Additionally, we find that there is a critical fluid Reynolds number above which the dynamo no longer saturates at or close to equipartition, but at a lower level characteristic of turbulent astrophysical dynamos. In terms of energy spectra there are marked differences between the high and low Re cases, but in physical space we identify some individual common processes that are likely to be a major part of the dynamo mechanism. Our intention was to illustrate that studying dynamo action by these kind of flows is a viable way to understand astrophysical dynamos. In a forthcoming paper we will follow up with a broad analysis in physical space of the numerical experiments presented here. We believe that the key to understanding the nature of non-linear dynamo action is to answer such questions as "where do the magnetic field lines come from, and where do they go?"

\section{References}

Archontis, V., Dorch, S. B. F., and Nordlund, Å.: 2003a, Astron. Astrophys. 397, 393.

Archontis, V., Dorch, S. B. F., and Nordlund, Å.: 2003b, Astron. Astrophys. 410, 759.

Cattaneo, F., Hughes, D. W., and Kim, E.: 1996, Phys. Rev. Lett. 76, 2057.

Childress, S. and Gilbert, A. D.: 1995, Stretch, Twist, Fold: The Fast Dynamo, Springer-Verlag, Berlin, p. 52.

Choudhuri, A. R.: 2003, Solar Phys. 215, 31.

Dorch, S. B. F.: 2000, Physica Scripta 61, 717.

Galloway, D. and Proctor, M.: 1992, Nature 356, 691.

Haugen, N. E. L., Brandenburg, A., and Dobler, W.: 2003, Astrophys. J. 597, L141.

Moreno-Insertis, F., Caligari, P., and Schüssler, M.: 1994, Solar Phys. 153, 449.

Nordlund, A., Brandenburg, A., Jennings, R. L., Rieutord, M., Roukolainen, J., Stein, R. F., and Tuominen, I.: 1992, Astrophys. J. 392, 647.

Parker, E. N.: 1979, Cosmical Magnetic Fields, Clarendon, Oxford.

Safier, P. N.: 1999, Astrophys. J. 510, L127.

Tanner, S. and Hughes, D.: 2003, Astrophys. J. 586, 585. 